\title{
Probabilistic Approach for Nondestructive Detection of Fatigue Crack Initiation and Sizing
}

\author{
Azadeh Keshtgar ${ }^{1}$, Mohammad Modarres ${ }^{2}$ \\ ${ }^{1,2}$ Center for Risk and Reliability, University of Maryland, College Park, MD 20742, USA \\ keshtgar@umd.edu \\ modarres@umd.edu
}

\begin{abstract}
${ }^{1}$ Early detection of a growing crack is one of the concerns in structural integrity and can be used to predict the remaining useful life of a structure. Acoustic Emission (AE) is a nondestructive testing method with potential applications for locating and monitoring fatigue cracks. A novel AE signal analysis approach is proposed in this paper to detect crack initiation and assess small crack growth behavior. A probabilistic AE-based model for small fatigue cracks was developed and the uncertainties of the model were estimated. The outcome of this research can be used to evaluate the integrity of structures and assess structural health by estimating the probability density function of the length of detected cracks. This paper discusses the methodology used, experimental approach, results obtained and predictive models developed.
\end{abstract}

\section{INTRODUCTION}

In materials engineering, fatigue cracking occurs when a material is subjected to cyclic loading. Structures such as bridges and airframes can be subjected to wide ranges of loading conditions during their operations. Extreme repeated loads may cause initiation of cracks and crack growth during the life of a structure.

A rich array of recent research in the literature deals with the probabilistic approaches that combine Nondestructive Evaluation (NDE) methods for detecting materials damage, especially for evaluating fatigue failures. Most of these use information from detection methods other than the AErelated NDE methods. Important recent NDE studies

Azadeh Keshtgar et al. This is an open-access article distributed under the terms of the Creative Commons Attribution 3.0 United States License, which permits unrestricted use, distribution, and reproduction in any medium, provided the original author and source are credited. include Chiachio, et al. (2014), Chiachio, et al. (2015) and Sankararaman and Mahadevan (2015) to name a few.

Several researchers have also focused on the study of small crack growth behavior and different small-crack test methods (Mc Dowell, 1997; Forth et al., 2005; Shyam et al., 2005; Kunkler et al., 2008); but no AE-based model for small crack growth have been offered. However, most of the success in correlating $\mathrm{AE}$ activities with crack growth has involved the latter stages of crack growth (Talebzadeh \& Robert, 2003; Rabiei \& Modarress, 2013; Keshtgar \& Modarres, 2013).

Some studies have been carried out to combine fracture toughness experiments with $\mathrm{AE}$ techniques in order to detect the damage initiation. Marquez and Olivares (1987) utilized an $\mathrm{AE}$ recording system to determine crack initiation and propagation at a thermally sprayed coating interface of nickel-chrome alloy with substrate of AISI 1045 steel. This study initiated an idea of relating abnormal behavior of AE signals to crack related events. However, it Chaswal et al., 2005 showed that $\mathrm{AE}$ amplitude is about an order of magnitude lower in Region I than in Region II due to lower $\Delta \mathrm{K}$ values. They investigated how low amplitude bursts in a short duration of time in region I correspond to micro-cleavage in thermally aged steel plates. Some recent studies hypothesized that a sudden and significant increase in $\mathrm{AE}$ events corresponds to damage initiation (Rahman et al., 2009; Elforjani \& Mba, 2009). Rahman, et al. (2009) offered that significant increase in hit count data might correspond to incipient damage due to wear in rolling elements during their contacts. Further, Mba (2009) presented result of their experimental investigation for detecting natural crack in slow speed shafts.

More recent studies (e.g., Han et al., 2014; Čapek et al., 2014; Vanniamparambil et al.,2015; and Mazal et al., 2015) report that much of the AE activity is recorded in the initial 
stages of damage accumulation compared to the later stages, and attribute this to material inclusions that crack in the early degradation periods. These studies hypothesize crack propagation rate based on the $\mathrm{AE}$ signals, but do not directly correlate them to crack initiation, nor do they consider associated crack detection probability and crack sizing errors using the detected $\mathrm{AE}$ features.

Despite a number of studies related to AE-based detection of fatigue damage in the literature, there has been no generalized approach for detection and sizing of crack initiation. Also, AE-based probabilistic prediction method for small crack initiation and growth is absent in the literature.

In this paper a new methodology of statistical AE data analysis for detecting crack initiation and developing a probabilistic AE model of small crack growth is introduced. The developed model can be used for detection of crack initiation as well as assessment of small crack growth behavior. This method can be used to assess the reliability of structures and evaluate health by estimating the probability of structure failure at a specified number of cycles, including associated uncertainties.

\subsection{The Basic Theory of AE}

Acoustic Emission is defined as a transient elastic wave generated by the rapid release of energy within a material and an AE signal is the electrical signal produced by a sensor in response to this wave (Chaswal et al., 2005; Rahman et al., 2009). An example of a typical AE waveform and its features is shown in Figure 1. In addition to recording the number of acoustic events and correlating this number to the level of damage (Eberhardt et al., 2005), it is also possible to record certain features of the $\mathrm{AE}$ waveforms. Some features are defined with respect to the specified threshold limit. These waveform features (AE events) include but not limited to the ones listed below (Beattie, 1983; Berkovits and Fang, 1995):

- Counts: Also known as "ring down count" is defined as the number of times that the AE signal amplitude exceeds a predefined subjective threshold value.

- Amplitude: The AE amplitude is the largest voltage peak in the waveform signal.

- Energy: AE energy is the measured area under the rectified signal envelope.

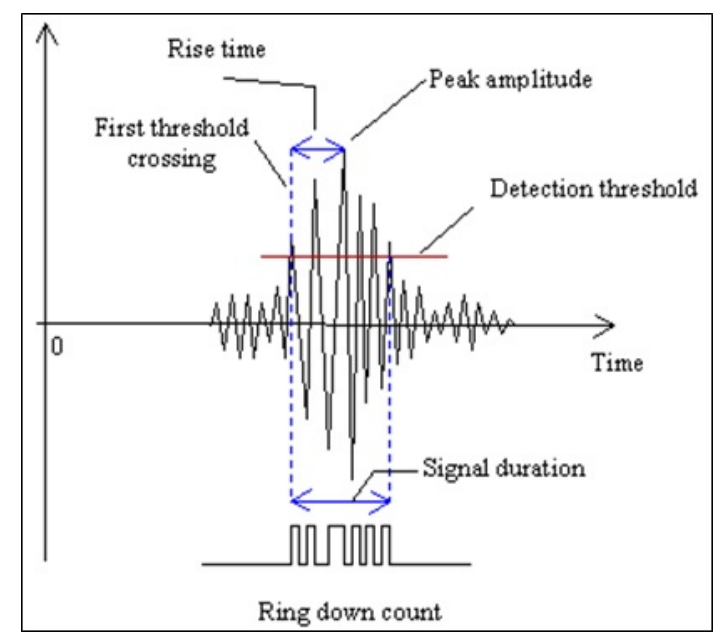

Figure 1. Typical AE signal and its characteristics (Kappatos \& Dermatas 2007)

\subsection{Fatigue crack initiation and small crack}

There is no universally accepted definition for fatigueinduced "small crack" and "crack initiation". Most experts consider cracks less than $1 \mathrm{~mm}$ in length as small (Larsen \& Allison, 1992; Anderson, 1995). Fatigue crack initiation is a subjective notion as well. Some consider crack initiation as corresponding with a fatigue phenomenon and some associate it with an arbitrarily specified crack length. For example, the U.S. Navy defines the presence of a crack of $250 \mu \mathrm{m}$ in length, as the point where crack initiation occurs (Iyyer et al., 2007; Papazian et al., 2009). Furthermore, others consider a crack length ranging from a size of grain diameter to about $100 \mu \mathrm{m}$ as crack initiation length depending on material and scale of interest. However, ranges of values have been used for identification of crack initiation in different materials within the literature (e.g., 51 $\mu \mathrm{m}$ for carbon steel, $120 \mu \mathrm{m}$ for BS250A53 steel and $1 \mathrm{~mm}$ for En7A steel (Bhattacharya \& Ellingwood, 1998) and up to $500 \mu \mathrm{m}$ for aluminum (Pearson, 1975)).

In this paper, a subjective fatigue crack initiation length was used for A17075-T6. This selection was primarily made based on the limitations in the crack size measurement method (i.e., optical microscopy) used in this study rather than a specific fatigue phenomenon. As such this study uses a subjective crack length of $50 \mu \mathrm{m}$ as the point of crack initiation. It is determined, however, that this crack initiation length is about a quarter of the average grain diameter for Al7075. It has been shown that approximately a $1-\mathrm{mm}^{3}$ of the above-mentioned material contains 700 grains (Papazian et al., 2009). Assuming spherical geometry of grains, the volume of each grain is about $1.43 \times 10^{-3} \mathrm{~mm}^{3}$ and the diameter of each grain can be estimated as $0.140 \mathrm{~mm}$ (140 $\mu m)$. Moreover, the applied optical crack measurement method carries some limitations for sizing of smaller cracks. More details on the methodology and its limitation are discussed in Sections 3.3.1 and 3.3. 


\section{EXPERIMENTAL SET UP}

To study the relationship between small crack length and the resulting AE signal, a group of standard experiments was performed under a controlled loading condition.

\subsection{Specimen}

Since in this research the observation of small crack length was done through optical microscopy, a series of standard flat dog-bone test specimens with the capability of being used for small crack monitoring were developed based on the ASTM standard (Morton et al., 1973). The samples made of 7075-T6 Aluminum alloy. Figure 2 is a schematic and geometric specification of the standard flat dog-bone specimen used in this research.

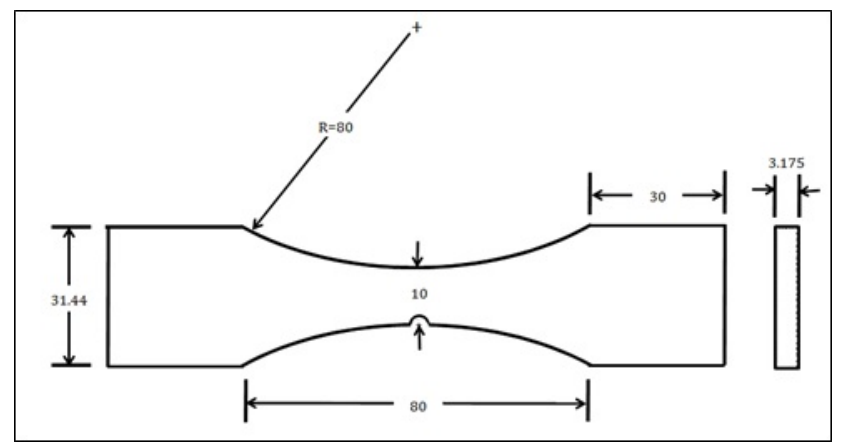

Figure 2. Drawing of flat dogbone samples (all dimensions in $\mathrm{mm}$ )

\subsection{Optical microscopy}

Fatigue cracks were monitored by direct measurement using an optical microscope on the front surface of the samples. The optical measurement system is comprised of several components: a high magnification optical microscope, a video camera attached to the microscope that records the crack growth path for the duration of the fatigue test, a dual arm fiber optic illuminator, a high resolution monitor, an image processing software with the time-lapse photography capability, and a micro-meter scale to calibrate the photographs taken. Figure 3 shows the optical microscopy test set up used for the small crack experiment.

\section{FAtigue testing}

In the experimental set up described above, eight fatigue specimens were tested at different loading conditions using both AE and optical microscopy measurement. Table 1 lists details of loading parameters for the tests performed.

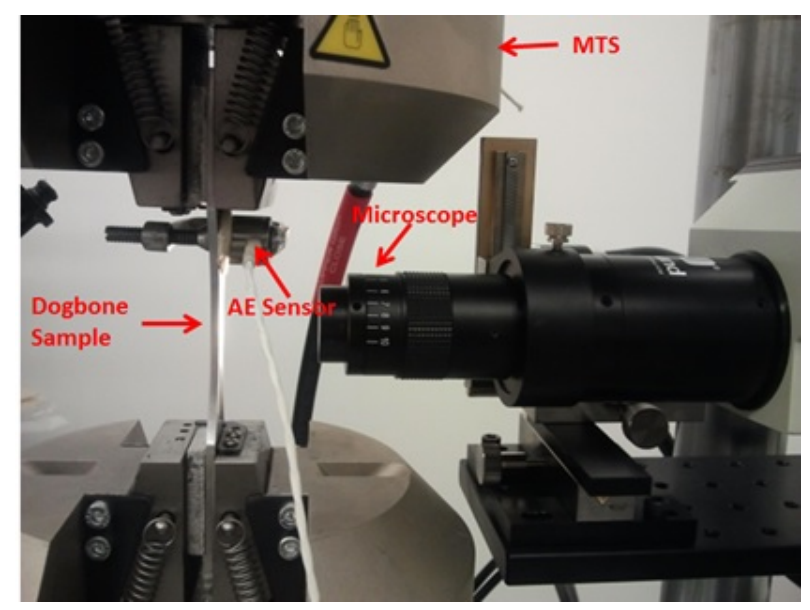

Figure 3. Test set up

\begin{tabular}{|c|c|c|c|}
\hline $\begin{array}{c}\text { Test } \\
\text { Reference }\end{array}$ & $\begin{array}{c}\text { Loading Frequency } \\
\text { (Hz) }\end{array}$ & $\begin{array}{c}\text { Loading Ratio } \\
\text { (R) }\end{array}$ & $\begin{array}{c}\text { Force (min-max) } \\
\text { KN }\end{array}$ \\
\hline T1 & 3 & 0.1 & $0.8-8$ \\
T2 & 3 & 0.08 & $0.6-8$ \\
T3 & 3 & 0.1 & $0.8-8$ \\
T4 & 2 & 0.5 & $6.5-13$ \\
T5 & 3 & 0.1 & $0.8-8$ \\
T6 & 2 & 0.3 & $3-10$ \\
T7 & 2 & 0.5 & $6.5-13$ \\
T8 & 2 & 0.3 & $3-10$ \\
\hline
\end{tabular}

Table 1. Details of loading parameters for all experiments

Acoustic Emission signals may be generated from a number of possible sources including background noise, micro-crack generation, or plastic deformation. In order to reduce uncertainties and determine the AE signals corresponding to crack growth, applying noise reduction techniques on the captured data is required. Noise reduction approaches are discussed in the following section.

\subsection{Noise reduction}

Various de-noising techniques have been proposed to filter AE signals due to crack growth (Morton et al., 1973; Peasron, 1975; ASTM, 2012). In the first step, the recorded AE data was filtered using the DiSP-4 source location software. Also, a dummy specimen was tested to determine the detection threshold below which operational background noise exist. This threshold allows for filtering the background noise and better capturing of crack-related signals.

It has also been observed that $\mathrm{AE}$ events occurring during the loading portion of a cycle are related to crack growth (Peasron, 1975; Wang et al., 1992; Robert \& Talebzadeh, 
2003). Therefore, the AE data taken during the loading portion of each cycle were used for data analysis. In addition, majority of researchers have assumed that only events occurring close to the maximum or peak load are associated directly with crack growth (Peasron, 1975; Georgiou, 2006; ASTM, 2012). So, the filtered AE events were separated for different percentages of the applied load range and it was determined that the $\mathrm{AE}$ events occurring within the top $20 \%$ of peak load shows the closest correlation with crack lengths (Rabiei \& Modarress, 2013).

\subsection{Crack Measurement}

The optical microscopy system with time-lapse photography was used for monitoring crack growth. The lengths of pictured cracks were measured using the Java-based imageprocessing software. Crack measurement was started before $50 \mu \mathrm{m}$ and continued until the crack length larger than 250 $\mu \mathrm{m}$ was observed. At this length the crack exceeds the U.S. Navy definition of crack initiation (Papazian et al., 2009) and the crack measurement was terminated.

\subsection{Experimental Uncertainties and Errors}

Crack measurement data may be uncertain in nature due to detection uncertainties and measurement errors associated with the optical measurement and sizing process. Estimation of crack lengths and consequently AE-model prediction can be affected by these uncertainties. In this section, experimental crack length measurement error is discussed and later used to quantify the uncertainty and validity of the developed AE model.

\subsubsection{Probability of detection (POD)}

Probability of detection expresses the probability of detecting a crack of a given length and is a common metric to assess the capability of a detection technique. A crack of a given length might be optically detected only at certain percentage of the time (out of the total number of tests) depending on factors such as sample subsurface cracks, specimen vibration, cyclic loadings, optical focus and human error. The POD for various crack lengths was calculated based on the hit-or-miss concept (Georgiou, 2006) as the ratio of the number of successful detections of a particular crack length over the total number of tests. POD increases with crack length and eventually attains a maximum value of unity at which all the cracks will be detectable. For this reason, the POD was estimated by a logistic function for this data to obtain a continuous POD curve. The cumulative distribution function of the loglogistic distribution is:

$$
F(a, m, s)=\frac{\exp \frac{\pi}{\sqrt{3}}\left[\frac{\log a-m}{s}\right]}{1+\exp \frac{\pi}{\sqrt{3}}\left[\frac{\log a-m}{s}\right]}
$$

where $m$ and $s$ are the parameters of the distribution and a denotes the crack length. The point estimates of the parameters of the logistic distribution are $m \approx 27 \mu m$ and $s \approx 9$ $\mu m$.

\subsubsection{Crack length measurement error}

Due to measurement errors, the experimental results are uncertain and do not exactly represent true values. The precision and accuracy of optical measurement tools, as well as the sizing techniques used to analyze and process the captured pictures of crack growth, contribute to measurement errors. This experimental measurement error is quantified by cross-validating the measurement results with known true crack lengths. To obtain a good estimation of the true crack lengths, a microscopy technique was used. When cracked, the specimen was removed from the MTS machine and was transferred to the optiphot microscope in Modern Engineering Material Instructional Lab (MEMIL) at the University of Maryland. This fixed stage microscope is capable of microflex photomicrography with a HFX camera. Using this setting, the final crack length at which the test was stopped was evaluated. An image processing tool was used to capture very high quality pictures of the crack at the various magnification levels. At each level of magnification, calibration was done by using a scaled ruler. The length of crack was measured later using the captured photographs. The measurements from this optiphot microscope were considered as the true crack lengths and were compared later with the measured crack lengths during online monitoring of the test.

Measurement error can then be expressed as a function of measured crack length. To do so, a multiplicative error model was used for estimation of measurement error. Based on this methodology, experimentally measured crack lengths are considered to be estimations and representation of the true crack length, given some error as it is shown in Eq. (2):

$$
\frac{a_{t, i}}{a_{e, i}}=F_{e, i} ; \quad F_{e} \sim L N\left(b_{e}, s_{e}\right)
$$

where $a_{t, i}$ is the true value of crack length, $a_{e, i}$ indicates the experimental measurement results and $F_{e}$ is the multiplicative error (both random and systematic) of experimental measurement with respect to true value. The lognormal distribution representing the uncertainty of the multiplicative error has parameters $b_{e}$ which is the mean of experimental measurement error, as well as $s_{e}$ which is the 
standard deviation of the experimental measurement error and both estimated using a Bayesian framework. For the details of the implemented Bayesian framework refer to section 4.2. The summary statistics for the marginal posterior pdf of parameters $b_{e}$ and $s_{e}$ as well as the distribution of $F_{e}$ are presented in Table 2 .

Experimental measurement error bounds can be determined from the 2.5 and 97.5 percentile of the multiplicative error of $\mathrm{Fe}$. The resulting upper bound was calculated as $23 \%$ while the lower bound is $-14 \%$. These results are presented graphically in Figure 4 . It can be noticed that there is a very slight positive systematic error (bias) from the true value (because the mean $F_{e}$ estimate slightly exceeds unity).

\begin{tabular}{|cllccc|}
\hline $\begin{array}{c}\text { Para- } \\
\text { meter }\end{array}$ & Mean & $\begin{array}{c}\text { Standard } \\
\text { Deviation }\end{array}$ & $2.5 \%$ & Median & $97.5 \%$ \\
\hline $\boldsymbol{b}_{\boldsymbol{e}}$ & 0.027 & 0.02678 & -0.025 & 0.0267 & 0.080 \\
$\boldsymbol{s}_{\boldsymbol{e}}$ & 0.0818 & 0.0232 & 0.0506 & 0.0774 & 0.138 \\
$\boldsymbol{F}_{\boldsymbol{e}}$ & 1.031 & 0.09282 & 0.8584 & 1.026 & 1.236 \\
\hline
\end{tabular}

Table 2. Estimated parameters of measurement error

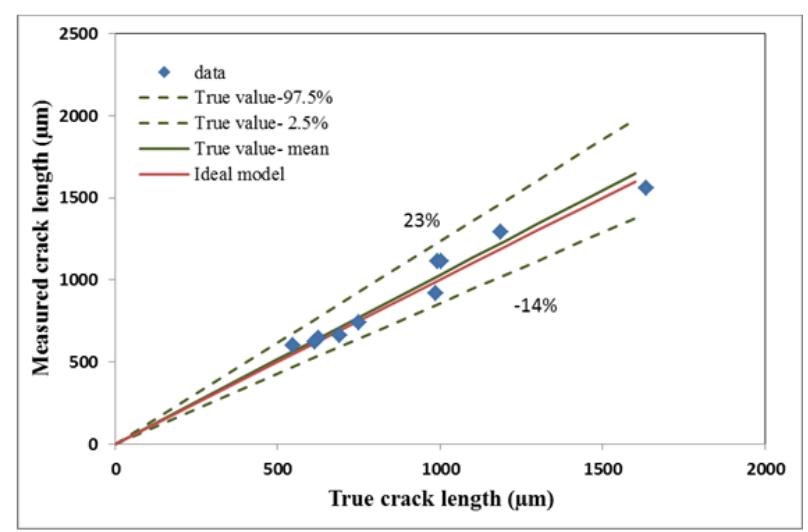

Figure 4. Experimental measurement error

The estimated parameters of the multiplicative measurement error model listed in Table 2 were used as the prior knowledge of the crack length measurement error in the Bayesian framework provided for the probabilistic model validation approach. The implemented approach considers both uncertainties in the POD and measurement errors while providing a framework for updating the probability distribution of the model parameters when new data becomes available.

\subsection{Acoustic Emission results}

\subsubsection{Acoustic Emission Counts}

After post processing of the recorded AE signals, the number of cumulative counts was calculated and plotted versus loading cycles. Results show that cumulative $\mathrm{AE}$ counts have considerable correlation with the measured crack lengths. A linear model can be developed based on this relation as shown in Figure 5.

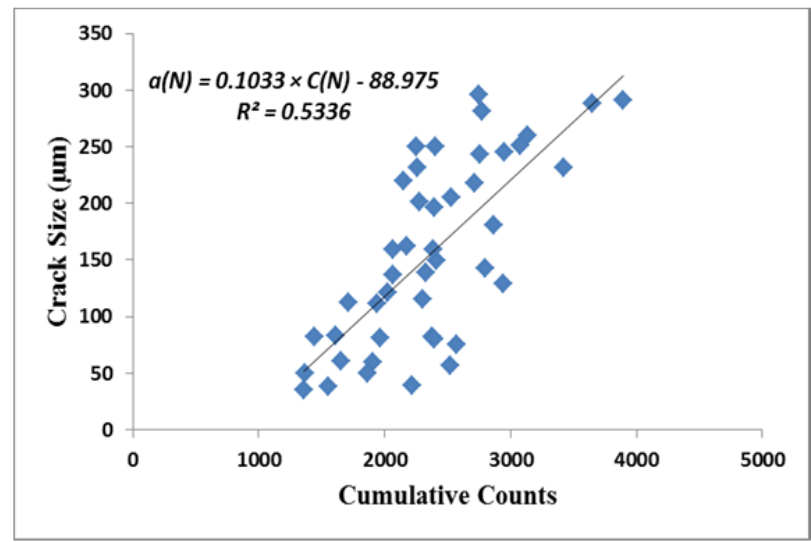

Figure 5. Correlation of Cumulative counts and crack length

\subsubsection{Acoustic Emission Intensity}

In order to provide a more effective AE measure of damage, a new approach was developed which employs multiple features of the $\mathrm{AE}$ signal and provides a measure for strength of signals. The main idea is that larger cracks produce stronger $\mathrm{AE}$ signals and not only the total number of counts, but also with the amplitude level of the $\mathrm{AE}$ signal, can quantify this strength. Therefore, estimation of small crack length can be implemented by simultaneous evaluation of these $\mathrm{AE}$ features. Based on this idea, a multiplicative correction factor was applied on the acquired counts using the observed amplitude of the signal. This correction factor was defined by the ratio of amplitude over the average or benchmark amplitude of signals.

A new $\mathrm{AE}$ index called AE-Intensity was proposed to combine $\mathrm{AE}$ counts and amplitude for more effective monitoring of damage state. AE-Intensity is a measure of signal strength and it was shown to have a better correlation with crack lengths. The intensity of AE signals is defined by Eq. (3):

$$
A E_{-} \text {Intensity }: \quad I(t)=C(t) \times \frac{A(t)}{A_{0}}
$$


where $C(t)$ is the cumulative counts at a specific time t, $A(t)$ indicates the amplitude of the signal and $A_{0}$ is the amplitude threshold.

The linear correlation between crack lengths and AEIntensity is shown in Figure 6. It can be seen that applying the amplitude correction factor to AE count considerably reduces the data scatter in comparison with cumulative counts. Based on the observed relationship, a linear model was proposed which uses AE-intensity as an independent variable and the crack length as dependent variable. The proposed model is introduced in the following section.

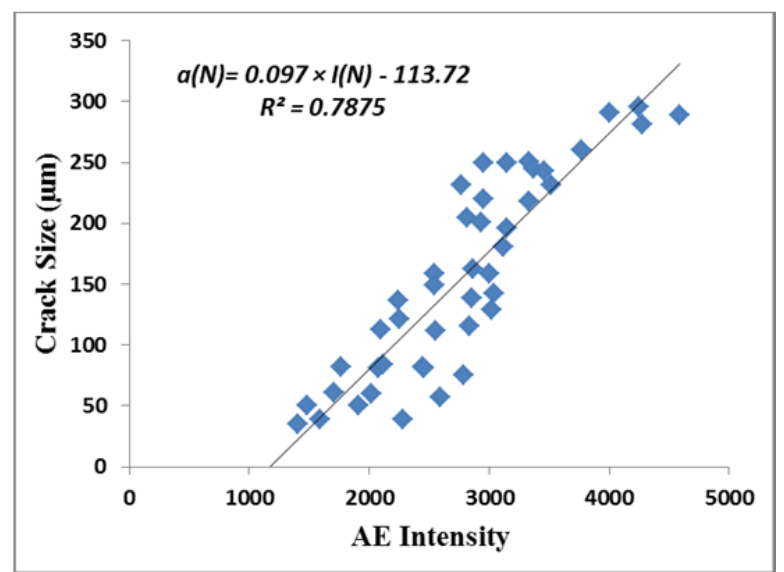

Figure 6. Correlation between intensity and crack length

\section{Probabilistic Model deVelopment}

The results of the analyzed experimental data were used to develop a probabilistic linear model for the estimation of the small crack length as the dependent variable while $\mathrm{AE}$ intensity is considered to be the independent variable.

\subsection{Modeling}

According to the observed correlation between AE intensity and crack length, a linear relationship was proposed in the following form:

$$
a(N)=\alpha \cdot I(N)+\beta
$$

where $a(N)$ indicates the true length of the small crack after $N$ loading cycles, $I(N)$ is the calculated AE intensity at cycle $N, \alpha$ and $\beta$ are the unknown model parameters. In this analysis, true crack lengths were used to evaluate the parameters of the proposed model.

After the first steps of analyzing the experimental data, results were used to estimate the unknown parameters in the proposed model (Eq. (4)) using least square regression analysis. Results are shown in Figure 7.

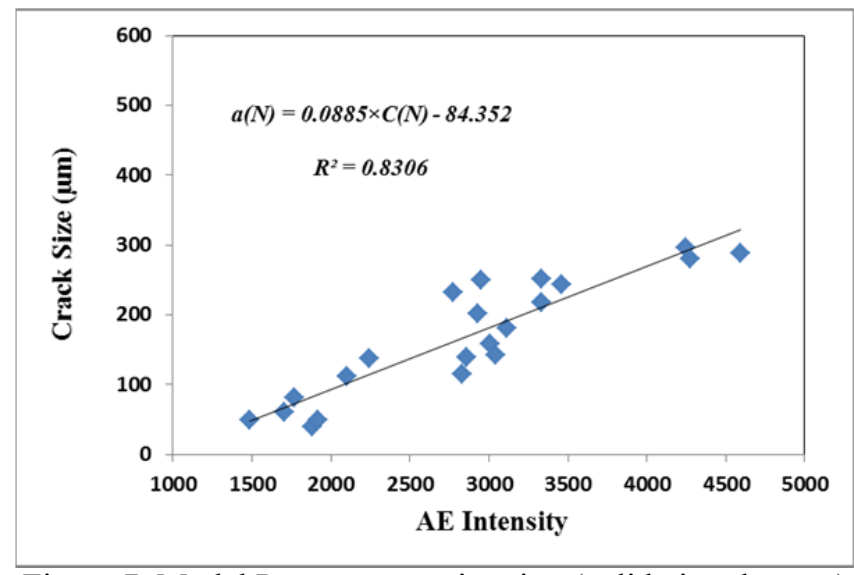

Figure 7. Model Parameters estimation (validation data set)

\subsection{Model validation and error estimation}

In order to validate the developed $\mathrm{AE}$ model, model predictions of small crack length were compared against the validation experimental data set. For a given value of $\mathrm{AE}$ Intensity, a prediction of crack length was estimated based on the developed AE-based model. The prediction results are then compared against the true crack lengths obtained by five validation experiments.

Model validation approach was originally developed by Ontivero, Cartillier and Modarres (2010) to account for uncertainties in fire model simulation predictions. In this Bayesian methodology, both model prediction and experimental results are considered to be estimations and representation of the true values, given some error as it is shown in Eqs. (2) and (5). The multiplicative error of experimental measurement with respect to the true value was introduced by Eq. (2) and the multiplicative error of model prediction with respect to true value is shown in Eq. (5):

$$
\frac{a_{t, i}}{a_{m, i}}=F_{m, i} ; F_{m} \sim L N\left(b_{m}, s_{m}\right)
$$

where $a_{t, i}$ is the true value, $a_{m, i}$ is the model prediction and $F_{m}$ is the multiplicative (fractional) error of the model prediction, with respect to the true value. Parameter $b_{m}$ is the mean (multiplicative) error of the model and $s_{m}$ is the standard deviation of the error. Accordingly, the multiplicative error of the measurement with respect to the model prediction could be defined by Eq. (6):

$$
\frac{a_{e, i}}{a_{m, i}}=\frac{F_{m, i}}{F_{e, i}}=F_{t, i}
$$

where $F_{t}$ is the multiplicative error of the model vs. measurement. Since both $F_{m, i}$ and $F_{e, i}$ distributions are lognormal, the distribution of $F_{t, i}$ would also be lognormal with mean and standard deviation of $\left(b_{m}-b_{e}\right)$ and 
$\sqrt{s_{m}^{2}+s_{e}^{2}}$, respectively. The Bayesian methodology used in this paper quantifies the uncertainties associated with the POD and crack length measurement error into the calculations. The Bayesian approach mathematically combines prior knowledge of crack length with uncertain experimental data and considers the systematic and random measurement errors and associated uncertainties, to estimate the posterior distribution of crack length. For more information about this approach and the concept of Bayesian uncertainty analysis see the paper by Ontiveros, et al. (2010). In this approach, the combined effect of the evidence used: POD data, measurement errors, and measured crack lengths were captured by a likelihood function. Considering Eqs. (2) and (5). The likelihood of the observed data using combined POD, measurement errors, measured and model-estimated crack is:

$L\left(\frac{a_{e, i}}{a_{m, i}}, b_{e}, s_{e} \mid b_{m}, s_{m}, m, s\right)=$

$\prod_{i=1}^{n}\left(\left(\operatorname{POD}\left(a_{e, i} \mid m, s\right)\right)\left(\frac{1}{\sqrt{2 \pi}\left(\frac{a_{e, i}}{a_{m, i}}\right) \sqrt{s_{m}^{2}+s_{e}^{2}}}\right) *\right.$

$\left.\exp \left(\frac{-\left[\ln \left(\frac{a_{e, i}}{a_{m, i}}\right)-\left(b_{m}-b_{e}\right)\right]^{2}}{2\left(s_{m}^{2}+s_{e}^{2}\right)}\right)\right) L\left(\frac{a_{e, i}}{a_{m, i}}, b_{e}, s_{e} \mid b_{m}, s_{m}, m, s\right)=$

$\prod_{i=1}^{n}\left(\left(\operatorname{POD}\left(a_{e, i} \mid m, s\right)\right)\left(\frac{1}{\sqrt{2 \pi}\left(\frac{a_{e, i}}{a_{m, i}}\right) \sqrt{s_{m}^{2}+s_{e}^{2}}}\right) *\right.$

$\left.\exp \left(\frac{-\left[\ln \left(\frac{a_{e, i}}{a_{m, i}}\right)-\left(b_{m}-b_{e}\right)\right]^{2}}{2\left(s_{m}^{2}+s_{e}^{2}\right)}\right)\right)$

where, the $P O D$ function is assumed to follow a log-logistic form based on Eq. (1). The parameters $b_{e}$ which is the mean of experimental measurement error, as well as $s_{e}$ which is the standard deviation of the experimental measurement error were previously estimated as discussed in Section 3.3. The summary statistics for the marginal posterior $p d f$ of parameters $b_{m}$ and $s_{m}$ as well as the distribution of $F_{m}$ are presented in Table 3.

\begin{tabular}{|cccccc|}
\hline $\begin{array}{c}\text { Para- } \\
\text { meter }\end{array}$ & Mean & $\begin{array}{c}\text { Standard } \\
\text { deviation }\end{array}$ & $2.5 \%$ & Median & $97.5 \%$ \\
\hline$b_{m}$ & -0.057 & 0.029 & -0.115 & -0.057 & 0.0002 \\
$S_{m}$ & 0.217 & 0.023 & 0.176 & 0.216 & 0.267 \\
$F_{m}$ & 0.968 & 0.218 & 0.609 & 0.944 & 1.465 \\
\hline
\end{tabular}

Table 3. Multiplicative error statistic summary
Model uncertainty bounds for the crack length estimation can be determined from the 2.5 and 97.5 percentiles of the multiplicative error of $\mathrm{Fm}$. The resulting upper bound was calculated as $46 \%$ while the lower bound is $-39 \%$. These results are presented graphically in Figure 8 . It can be noticed that there is a very slight systematic errors (bias) from the true value in the results showing that the model slightly overestimates the true length of the crack.

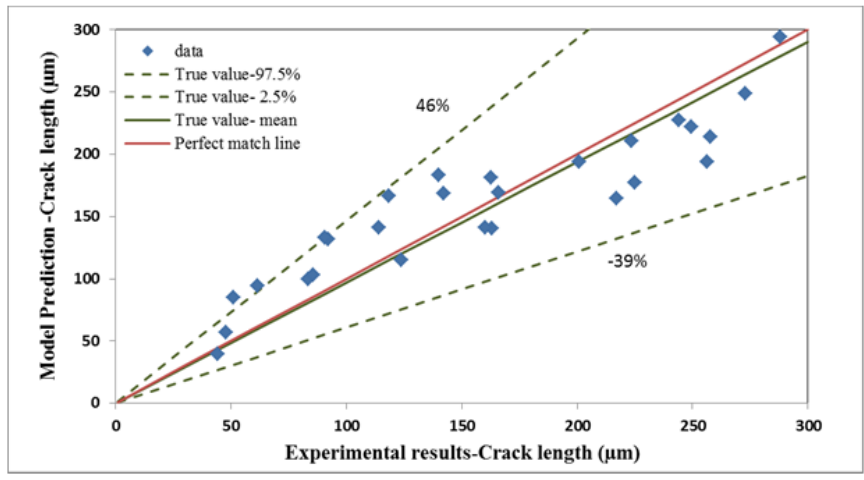

Figure 8. Comparison of $\mathrm{AE}$ model prediction and experimental results.

Assuming $a_{m}$ is the model prediction of crack length, the true crack length model prediction then can be estimated by multiplying the distribution of $a_{m}$ by $F_{m}$ :

$$
a_{t}=F_{m} \cdot a_{m}
$$

which can be estimated by a lognormal distribution as Eq. (10).

$$
a_{t} \sim L N\left(\ln \left(a_{m}\right)+b_{m}, s_{m}\right)
$$

The model prediction results were modified using the resulted bias distribution. Figure 9 illustrates the model prediction uncertainty bounds as well as the modified prediction results. As it can be seen, the developed model slightly over predicts the crack lengths. It can be seen from the differences between the mean values of model prediction and the true values in Figure 9. This over prediction result results in a slightly conservative model which can be used to predict a critical crack before it happens. 


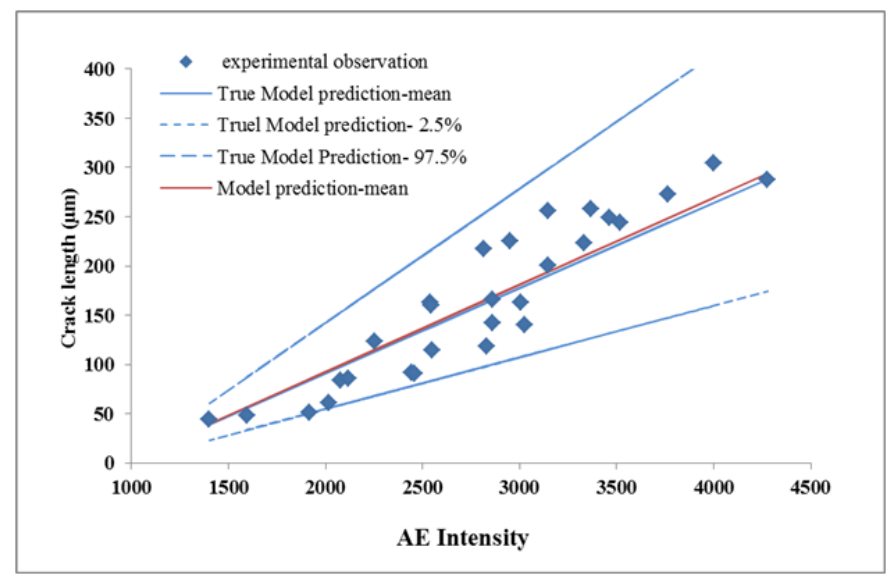

Figure 9. Model Prediction with multiplicative error

The results show that the developed AE-model reasonably estimates the true crack length. There is a small bias in prediction that can be accounted for with the inclusion of the model error factor, $F_{m}$, which allows for an estimation of the true crack lengths. The resulting model can be used to appropriately estimate the true crack length distribution, without a bias.

\section{Conclusion}

A novel methodology for in-situ monitoring of small fatigue crack initiation and growth using AE signal processing technique was introduced. Investigation of the uniform cyclic loading tests on A17075-T6 specimens indicated that initiation of cracks smaller than the grain size could be identified using statistical analysis of the resulting AE signals. Several standard fatigue tests were performed using flat dog-bone specimens.

Acoustic Emission data acquisition was used in conjunction with optical microscopy for online monitoring of crack length. The data captured was used to establish a correlation between certain $\mathrm{AE}$ signal features and the measured crack length. Also a probabilistic model of fatigue crack length distribution based on a combination of $\mathrm{AE}$ signal characteristics including probability of crack detection and measurement error was developed.

The proposed model was validated and proved to be effective for detection of crack initiation as well as prediction of small crack growth in early stages of propagation. Development of the proposed AE monitoring technique reported in this paper facilitates early detection of fatigue crack, allows for the prognostics and life predictions of the structure. It should be noted that the implementation of the developed model is limited to A17075-T6 specimens with the specific geometry highlighted in Figure 2. The variability of the results with respect to different materials and different geometries should be reviewed in the future works. More suggestions for potential future work are highlighted below:

- The experimental data used in this research were obtained through standard fatigue tests with constant amplitude loadings. It's recommended to implement a set of experiments to review the effect of random amplitude loading profile on the developed AE model.

- The scope of this research does not include addressing challenges of implementing the developed model for different materials and different geometries. It would be desirable to obtain data for samples made of different geometries and materials and study the applicability of the proposed AE model for some commonly used structures.

- In this research the location of crack initiation was assumed to be known since the standard specimen was used for experiments. However, there are some techniques suggested in the literature to locate the crack using multiple AE sensors. For instance, one of the proposed methods is triangulation which can be used to spot the location of AE source. This method can be applied as an extension of the current work to further enhance the capability of the proposed model for potential industrial applications.

\section{REFERENCES}

Anderson, T., (1995). Fracture Mechanics: Fundamentals and Applications. Boca Raton: Boca CRC Press.

ASTM E466 (2012). Standard practice for conducting force controlled constant amplitude axial fatigue tests of metallic materials. ASTM International, vol. i.

Beattie, A. G. (1983). Acoustic emission, principles and instrumentation. Journal of Acoustic Emission, 2, 95128.

Berkovits, A. \& Fang, D. (1995). Study of fatigue crack characteristics by acoustic emission. Engineering Fracture Mechanics, 51(3), 401-416.

Bhattacharya B. \& Ellingwood, B. (1998). Continum damage mechanics analysis of fatigue crack initiation, Journal of Fatigue, 20(9), 631-639.

Capek J., Mathis, K., Clausen, B., Straska, J., Beran, P. and Lukas, P. (2014). Study of the loading mode dependence of the twinning in random textured cast magnesium by acoustic emission and neutron diffraction methods. Materials Science \& Engineering, 602, 25-32.

Chaswal, V., Sasikala, G., Ray, S. K., Mannan, S. L. \& Raj, B. (2005). Fatigue crack growth mechanism in aged 9Cr-1Mo steel: threshold and Paris regimes. Materials Science and Engineering: A, 395(1-2), 251-264.

Chiachio, J., Chiachio, M., Sankararaman, S., Saxena, A. \& Goebel, K. (2015). Condition-based prediction of time- 
dependent reliability in composites. Reliab Eng Syst Safe, 142: 134-147.

Chiachio, M., Chiachio, J., Rus, G. \& Beck, J. L. (2014). Predicting fatigue damage in composites: a Bayesian framework. Struct Saf, 51, 57-68.

Eberhardt, E., Stead, D., Stimpson, B. \& Read, R. S. (1997). Changes in Acoustic event properties with progressive fracture damage. International journal of Rock Mech. \& Min. Science, 34(71), 3-4.

Elforjani M. \& Mba, D. (2009). Natural mechanical degradation measurements in slow speed bearings. Engineering Failure Analysis, 16(1), 521-532.

Forth, S. C., Newman, J. C. \& Forman, R. G. (2005). Evaluation of Fatigue Crack Thresholds Using Various Experimental Methods. Journal of ASTM International, 2(6), 1-16.

Georgiou, G. A. (2006). Probability of Detection (PoD) curves: Derivation, applications and limitations. Jacobi Consulting Limited, Research Report 454.

Han Z., Luo, H., Sun, C., Li, J., Papaelias, M. \& Davis, C. (2014). Acoustic emission study of fatigue crack propagation in extruded AZ31 magnesium alloy. Materials Science \& Engineering, 597, 270-278.

Iyyer, N., Sarkar, S., Merrill, R. \& Phan, N. (2007). Aircraft life management using crack initiation and crack growth models - P-3C Aircraft experience. International Journal of Fatigue, 29(9-11), 584-1607.

Kappatos, V. \& Dermatas, E. (2007). Crack detection in noisy environment including raining conditions. Aircraft Engineering and Aerospace Technology, 79(2),163-169.

Keshtgar, A. \& Modarress, M. (2013). Acoustic EmissionBased Fatigue Crack Growth Prediction. Reliability and Maintainability Symposium (1-5), Jan. 28-31, Orlando, FL.

Künkler, B, Düber,O, Köster, P, Krupp, U, Fritzen, C. P., and Christ, H. J. (2008). Modelling of short crack propagation-Transition from stage I to stage II. Engineering Fracture Mechanics, 75(3-4), 715-725.

Larsen, J. M. \& Allison, J. E. (1992). Small Crack Test Methods. American Society for Testing and Materials.

Marquez, J. G. M. \& Olivares, J. L. (1987). A study of crack initiation and propagation in Ni-Cr thermally sprayed coatings using Acoustic Emission techniques. Thin Solid Films, 153, 243-252.

Mazal, P. Vlasic, F. \& Koula, V. (2015). Use of acoustic emission method for identification of fatigue microcracks creation. Procedia Engineering, 133, 379-388.

McDowell, D. L. (1997). An engineering model for propagation of small cracks in fatigue. Engineering Fracture Mechanics, 56(3), 357-377.

Miller, R. K., Hill, E. V. K. \& Moore, P. O. (2005). Acoustic Emission Testing. Nondestructive Testing Handbook, ASNT.
Morton, T. M., Harrington, R. M. \& Bjeletich, J. G. (1973). Acoustic Emission of fatigue crack growth. Engineering Fracture Mechanics, 5, 691-697.

Ontiveros, V., Cartillier, A. \& Modarres, M. (2010) An Integrated Methodology for Assessing Fire Simulation Code Uncertainty. Nuclear Science and Engineering, 166(3), 179-201.

Papazian, J. M., Anagnostou, E. L., Engel, S. J., Hoitsma, D., Madsen, J., Silberstein, R. P., Welsh, G. \& Whiteside, J. B. (2009). A structural integrity prognosis system. Engineering Fracture Mechanics, 76(5), 620632.

Pearson, S. (1975). Initiation of fatigue cracks in commercial aluminium alloys and the subsequent propagation of very short cracks. Engineering Fracture Mechanics, 7, 235-247.

Physical Acoustic Corporation (2007). AEwin Software User's Manual, Princeton Junction, NJ.

Rabiei, M. \& Modarress, M. (2013). Quantitative methods for structural health management using in situ acoustic emission monitoring. International Journal of Fatigue, 49, 81-89.

Rahman, Z., Ohba, H., Yoshioka, T. \& Yamamoto, T. (2009). Incipient damage detection and its propagation monitoring of rolling contact fatigue by acoustic emission. Tribology International, vol. 42, no. 6, pp. 807-815, Jun. 2009.

Roberts, T. M. \& Talebzadeh, M. (2003). Fatigue life prediction based on crack propagation and acoustic emission count rates. Journal of Constructional Steel Research, 59(6), 679-694.

Sankararaman, S. \& Mahadevan, S., (2015). Statistical Approach to Structural Damage Diagnosis under uncertainty. Emerging Design solutions in Structural Health Monitoring Systems, 153-169.

Shyam, A., Allison, J. \& Jones, J. (2005). A small fatigue crack growth relationship and its application to cast aluminum. Acta Materialia, 53(5), 1499-1509.

Vanniamparambil, P. A. Guclu, U. and. Kontsos A. (2015). Identification of crack initiation in aluminum alloys using acoustic emission. Experimental Mechanics, 55(5),837-850.

Wang, Z. F. , Li, J., Ke, W. and Zhu, Z. (1992). Acoustic emission monitoring of fatigue crack closure. Scripta Metallurgica et Materialia, 27(12), 1691-1694.

\section{BIOGRAPHIES}

Azadeh Keshtgar is a Reliability engineer and has earned her PhD from the University of Maryland (UMD), College Park, USA at 2013. She also earned a MSc degree in reliability engineering from UMD and another MSc before that at Control Engineering from the Petroleum University of Technology, Tehran, Iran. She graduated in Aerospace Engineering (B.S. degree) from Sharif University of Technology, Tehran, Iran (2002). She worked as a research 
and teaching assistant at the Center for Risk and Reliability, University of Maryland and is currently working at TDA, Inc. as a senior Reliability engineer. She has worked extensively in uncertainty modeling and analysis, risk and reliability estimation, accelerated life testing, fracture mechanics and aerospace structural integrity.

Mohammad Modarres is the Director of Reliability Engineering and the Nicole Y. Kim Eminent Professor of Engineering in the University of Maryland, College Park, United States. He specializes in probabilistic risk assessment and management, uncertainty analysis, probabilistic physics of failure and probabilistic fracture mechanics modeling. He earned his B.S. degree from Tehran Polytechnic in mechanical engineering, a Master's degree from Massachusetts Institute of Technology in mechanical engineering and a $\mathrm{PhD}$ from Massachusetts Institute of Technology in nuclear engineering. He has served as a consultant or board member to several governmental agencies including the Nuclear Regulatory Commission, Department of Energy, National Academy of Sciences, and several national laboratories in areas related to nuclear safety, probabilistic risk assessment, probabilistic fracture mechanics and physics of failure. He has over 350 papers in archival journals and proceedings of conferences. He has published a number of textbooks, edited books and book chapters in various areas of nuclear safety, risk and reliability engineering. $\mathrm{He}$ is a University of Maryland Distinguished Scholar-Teacher and a fellow of the American Nuclear Society. 